\title{
HYPOTENSION, HYPONATREMIA, HYPERKALEMIA IN A PATIENT WITH CARCINOMA OF LUNG
}

\author{
By Faroque A. Khan, MB, FACP, FRCP (C), FCCP, \\ Sunil Mehra, MB, Arfa Khan, MB, \\ and \\ Robert Zorowitz, M.D.
}

DOI: http://dx.doi.org/10.5915/15-1-12379

\section{Case History}

In April 1982, a 60 year old black woman was diagnosed to have poorly differentiated large cell carcinoma of the right lung. (Fig. 1). She refused treatment and left the hospital against medical advice. Six weeks later she was readmitted with complaints of progressive weakness, anorexia and significant weight loss. The blood pressure was $80 / 60 \mathrm{mmHg}$ and temperature of $100.4^{\circ} \mathrm{F}$. The skin had poor turgor and dark pigmentation of lips and hands was noticed. She had poor muscle mass and was emaciated. Relevant laboratory findings included: serum sodium $128 \mathrm{~m} \mathrm{Eq/L}$ (135-145), serum potassium $6.1 \mathrm{~m} \mathrm{Eq/L} \mathrm{(3.5-5.0),} \mathrm{serum} \mathrm{chloride} 97$ $\mathrm{m} \mathrm{Eq} / \mathrm{L}$ (100-110), venous bicarbonate $15 \mathrm{~m} \mathrm{Eq/L}$ (24-32), BUN $18 \mathrm{mgm} / \mathrm{DL}(10-26)$, creatinine 1.4 mgm/DL (0.7-1.4). Het 24.8, WBC 23.5, polys 79 , bands 7 , lymph 6 , mono 3 , eosinophils $5 \%$. Liver function tests revealed increased alkaline phosphatase, but liver and spleen scan failed to confirm any metastasis. Bone scan was normal. In view of the hyponatremia, hyperkalemia, hypotension and the clinical features described, a specific diagnostic test was performed at this time.

\section{Metastatic Bronchogenic Carcinoma with Addison's Disease}

Clinically, a diagnosis of adrenal insufficiency was suspected and an abdominal Computerized Axial Tomographic (CAT) scan was performed. The scan was highly suggestive of bilateral adrenal metastases. (Fig. 2). She was given prednisone which led to gradual improvement in her blood chemistries and general well being. Two months later she died from metastatic bronchogenic carcinoma. No autospy was done.

Autoimmune destruction of the adrenals is now the commonest cause of primary adrenal hypofunc-

From the Division of Pulmonary Medicine, Department of Medicine, Queens Hospital Center Affiliation, Long Island Jewish-Hillside Medical Center, Queens, New York and School of Medicine, Health Sciences Center, State University of New York at Stony Brook tion or Addison's disease, other causes being metastatic carcinoma and tuberculosis. Clinical features of adrenal insufficiency develop when more than $90 \%$ of adrenal cortices are replaced by the tumor and metastatic carcinoma accounts for less than $1 \%$ of the reported cases of Addison's Disease. ${ }^{1}$ The reported autospy incidence of adrenal metastasis in various cancers varies from 8.6 to $27 \%$. The commonest cancer spreading to adrenal is lung $(64 \%)$ followed by breast, stomach and occasionally from uterus, pancreas, seminoma of testis, melanoma and adrenal carcinoma. ${ }^{2}$

Biochemical testing with ACTH and serum and urine cortisol levels have been employed to confirm the diagnosis in these patients. However, in spite of the frequent pathologic involvement of the adrenals with metastitic carcinoma the clinical diagnosis of

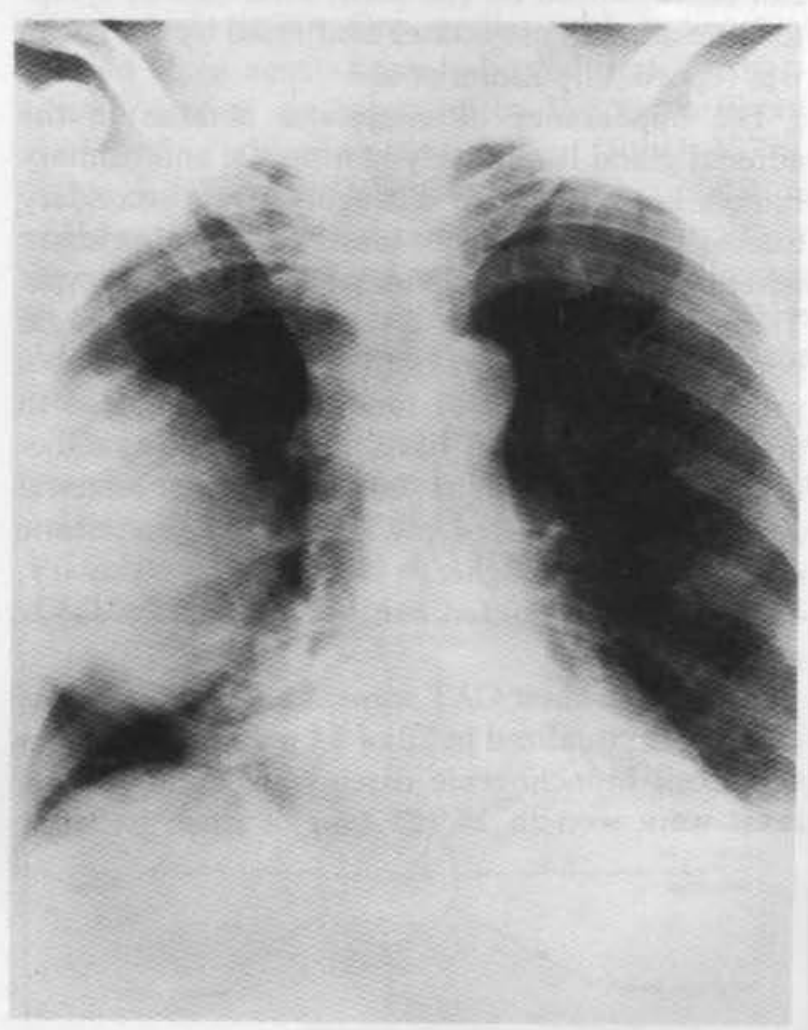

Figure 1: Postero-anterior view of the chest shows a large neoplasm in the right lung. 


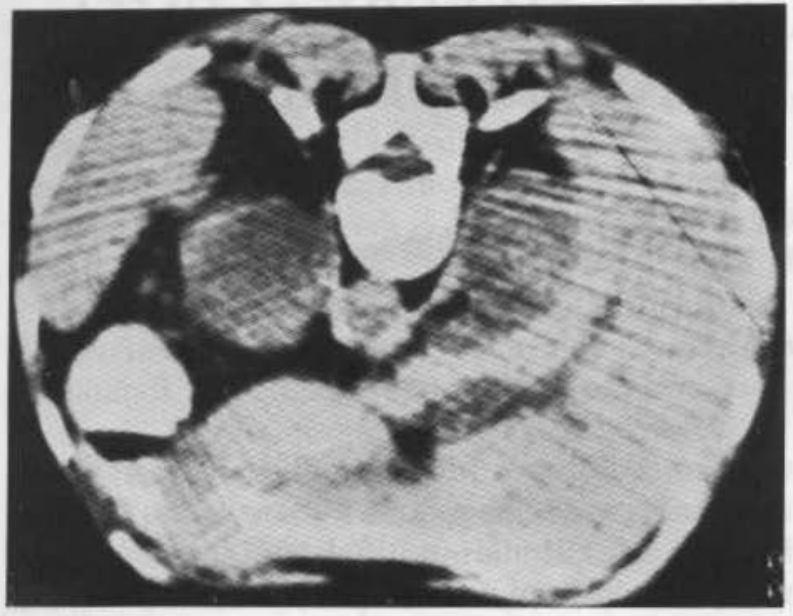

Figure 2: Computed axial tomogram of the upper abdomen shows bilateral large adrenal masses (Arrowheads) L-liver, S-spleen, A-aorta.

adrenal hypofunction is infrequently made, due perhaps to the similarity of symptoms of adrenal hypofunction and progressive maligancy. To date, only 10 cases of biochemically documented metastatic bronchogenic carcinoma to the adrenals causing clinical adrenal hypofunction have been reported. ${ }^{35}$ CAT scan is the most useful noninvasive method for visualizing the adrenals. With the newer third and fourth generation CAT scanners in more than 95 per cent of normals, adrenals can be visualized on the scan. First case of symptomatic adrenal metastases confirmed by CAT scan was reported by Zimm et al. ${ }^{6}$

The appearance of metastatic nodules in the adrenal gland is relatively nonspecific and primary tumors are difficult to distinguish from secondary tumors by CAT. The most common primary adrenal lesion is the adrenal cortical adenoma. These tumors occur in 2 to 3 percent of general population. ${ }^{7}$ However, in the presence of a known primary malignancy of lung, a mass detected in adrenal gland by CAT has a high probability of being metastatic in origin. In patients with bilateral adrenal masses, the probability of metastatic disease is even higher. ${ }^{8}$ If clinically necessary, histologic confirmation can be obtained by CATguided biopsy.

On routine chest CAT scans, at least one adrenal gland was visualized in 70 of 84 patients with nonsmall cell bronchogenic carcinoma. Solid adrenal mass were seen in $15(21.4 \%)$ of these patients, thereby altering the management approach of such patients. Thus, it seems appropriate that the adrenal glands should be routinely included while a CAT examination of the chest is being performed. ${ }^{9}$ With routine CAT scanning of the abdomen, fifteen percent of untreated patients with small cell carcinoma of the lung were found to have adrenal metastases. ${ }^{10}$

Thus, CAT scanning has assumed an important role in the staging of patients with lung cancer. During chest CAT scanning, the field should be enlarged to include the adrenals and up to $21 \%$ of unsuspected adrenal masses may be visualized thereby altering the treatment plan. In diagnosed cases of bronchogenic carcinoma, any clinical suspicion of adrenal hypofunction can be readily diagnosed by abdominal CAT scanning and replacement steroid therapy may offer palliation in such patients, as exemplified by our case report.

\section{References}

1. Guttman PH: Addison's Disease-A Statistical Analyses of 566 Cases and a Study of Pathology. Arch Pathol 10:742-745, 895-935, 1930.

2. Bullock WK, Hirst AE Jr.: Metastatic Carcinoma of the Adrenal Glands. Am J Med Science 225:521-524, 1953.

3. Sheridan CM: Adrenal Metastases Causing Addison's Disease. Br Med J 2:767-768, 1966.

4. Vieweg WVR, Reitz RE, Weinstein RL: Addison's Disease Secondary to Metastatic Carcinoma. Cancer 31:1240-1243, 1973.

5. Rosenthal FD, Davies MK, Burden AC: Malignant Disease Presenting as Addison's Disease. Br Med J 2:1591-1592, 1978.

6. Zimm S, Gardner DF, Walsh JW, et al.: Addison's Disease as the Sole Clinical Manifestation of Recurrent Bronchogenic Carcinoma. So Med J 74:1016-1018, Aug. 1981.

7. Thomas JL, Barnes PA, Bernardino ME, et al.: Diagnostic Approaches to Adrenal and Renal Metastases. Rad Clin N Am 20, No. 3, 531-544, Sept. 1982.

8. Zoronoza J, Bernardino ME.: Bilateral Adrenal Metastases. "Headlight" sign Urology, 15:91-92, 1980.

9. Nielsen ME, Heaston DK, Dunnick NR, Korobkin M: Preoperative CT Evaluation of Adrenal Glands in NonSmall Cell Bronchogenic Carcinoma. Am J Roent. 139:317-320, Aug. 1982.

10. Vas W, Zylak MD, Mather D, et al: The Value of Abdominal Computed Tomography in the Pre-treatment Assessment of Small Cell Carcinoma of Lung. Radiology 138:417-418, Feb. 1981.

Address correspondence to: F. Khan, Chief, Pulmonary Medicine, Queens Hospital Center, "B" Building-Room 320, 82-68 164th Street, Jamaica, New York 11432. 